\title{
Inferring black hole charge from backscattered electromagnetic radiation
}

\author{
Luís C. B. Crispino, ${ }^{1, *}$ Sam R. Dolan, ${ }^{2, \dagger}$ Atsushi Higuchi, ${ }^{3,+}$ and Ednilton S. de Oliveira ${ }^{1, \S}$ \\ ${ }^{1}$ Faculdade de Física, Universidade Federal do Pará, 66075-110 Belém, Pará, Brazil \\ ${ }^{2}$ Consortium for Fundamental Physics, School of Mathematics and Statistics, University of Sheffield, \\ Hicks Building, Hounsfield Road, Sheffield S3 7RH, United Kingdom \\ ${ }^{3}$ Department of Mathematics, University of York, Heslington, York YO10 5DD, United Kingdom
}

(Received 24 July 2014; published 15 September 2014)

\begin{abstract}
We compute the scattering cross section of Reissner-Nordström black holes for the case of an incident electromagnetic wave. We describe how scattering is affected by both the conversion of electromagnetic to gravitational radiation, and the parity dependence of phase shifts induced by the black hole charge. The latter effect creates a helicity-reversed scattering amplitude that is nonzero in the backward direction. We show that from the character of the electromagnetic wave scattered in the backward direction it is possible, in principle, to infer if a static black hole is charged.
\end{abstract}

DOI: 10.1103/PhysRevD.90.064027

PACS numbers: 04.40.-b, 04.70.-s, 11.80.-m

\section{INTRODUCTION}

In 1909, the Geiger-Marsden (GM) experiment [1] revealed the internal structure of the atom, demonstrating the existence of a compact nucleus $\sim 10^{4}$ times smaller in diameter than the atom itself. Later, recalling the anomalous backscattering of $\alpha$ particles from gold atoms, Rutherford remarked [2] that "it was almost as incredible as if you fired a 15-inch shell at a piece of tissue paper and it came back and hit you."

Here, we consider anomalous backscattering in a rather different setting. In principle, one may infer the internal structure of astrophysical systems harboring compact nuclei-such as black holes-in a way analogous to the GM experiment [(i)] by observing the scattering of waves and particles on the curved spacetimes of general relativity [(ii)]. Although a difference in scale prohibits laboratorybased experiments, there are many formal similarities. For instance, long-ranged $(1 / r)$ effects dominate, but reveal little about internal structure: in case (i) a Coulomb field generates Rutherford scattering, which is insensitive to internal structure, and in case (ii) the "Newtonian" field component leads to an analogous scattering pattern, with an Einstein ring [3] that is insensitive to the mass distribution. In either case, the "nucleus" attracts other matter/fields (i.e. electrons, accretion disks, etc.) which may screen, in case (i), or distort, in case (ii), the scattering pattern. The nucleus itself generates weak effects on a narrower scale: for example, direct collisions in (i) and, in (ii), excitement of neutron star resonances, or in the black hole case, absorption and a host of effects associated with the "light

\footnotetext{
*crispino@ufpa.br

†s.dolan@sheffield.ac.uk

atsushi.higuchi@york.ac.uk

§esdeoliveira@gmail.com
}

ring" of radius $\sim 3 G M / c^{2}$, where $G$ is Newton's constant and $M$ is the mass of the black hole, such as quasinormal ringing [4]. In particular, the light ring scatters flux through large angles, creating interference and a "glory" in the backward direction [5]. Thus, backscattered flux may provide telling hints about internal structure.

Several works have already been devoted to the topic of scattering by black holes (see Ref. [6] and references therein). Many interesting black hole phenomena were explored in the 1970s, such as superradiance [7], the glory effect [8], and Hawking radiation [9]. It was realized that charged black holes provide an efficient mechanism for the conversion of electromagnetic (EM) to gravitational radiation, and vice versa [10-14].

Here, we consider an EM wave incident upon a ReissnerNordström black hole. It was recently shown that the conversion mechanism plays an important role in absorption processes $[15,16]$, such that the gravitational and the EM absorption cross sections coincide in the extremal limit [17]. Here, we consider the scattering of EM flux. We show that charged black holes, unlike their Schwarzschild counterparts [18], can scatter EM flux through exactly $180^{\circ}$, leading to a distinctive signature.

The Reissner-Nordström line element is given by [19]

$$
d s^{2}=f(r) d t^{2}-f(r)^{-1} d r^{2}-r^{2}\left(d \theta^{2}+\sin ^{2} \theta d \phi^{2}\right),
$$

where $f(r)=\left(1-r_{+} / r\right)\left(1-r_{-} / r\right)$, with $\quad r_{ \pm}=M \pm$ $\sqrt{M^{2}-Q^{2}}$, and $M$ and $Q$ are the black hole mass and charge, respectively. We use natural units with $c=G=1$ and the metric signature $(+---)$ throughout.

It was shown in Refs. [20-23] that, at small scattering angles, the scattering cross section is

$$
\frac{d \sigma}{d \Omega} \approx \frac{16 M^{2}}{\theta^{4}}+\frac{3 \pi\left(5 M^{2}-Q^{2}\right)}{4 \theta^{3}}+O\left(\theta^{-2}\right) .
$$


Black hole charge leads to a subdominant correction to scattering in the weak-field region. Lensing properties, such as the Einstein ring, are dominated by the mass of the black hole, and the presence of charge is difficult to infer.

At larger scattering angles, the cross section will exhibit "orbiting" oscillations [24], due to interference between wave fronts which pass in opposite senses around the black hole. Such oscillations are signatures of the strong-field region of the spacetime. Near the backward direction $\left(\theta \sim 180^{\circ}\right)$, such interference typically creates a glory. For massless fields of spin $s$, a WKB analysis gives [5,25]

$$
\frac{d \sigma}{d \Omega}=\left.2 \pi \omega b_{g}^{2} \frac{d b}{d \theta}\right|_{b=b_{g}} J_{2 s}^{2}\left(\omega b_{g} \sin \theta\right) .
$$

Here, $b_{g}$ is the impact parameter associated with a null geodesic passing all the way around the black hole, near the light ring. Note that the Bessel function $J_{2 s}$ is 0 at $180^{\circ}$ for massless fields with spin $(s>0)$. Hence, according to Eq. (3) no flux will be scattered through exactly $180^{\circ}$. The semiclassical interpretation is that an annulus of the incident wave front is focused onto $\theta=180^{\circ}$ and, since the spin is parallel transported along each geodesic passing through the annulus, the circular symmetry results in completely destructive interference.

However, even in uncharged $(Q=0)$ black hole spacetimes, there is an additional feature in the backscattering of gravitational waves $(s=2)$ [26] that is not captured by Eq. (3). There arises a difference in the phase shift of the "odd- and even-parity" contributions (see below), which generates an additional scattering amplitude $\mathcal{G}(\theta)$ associated with the reversal of helicity [6]. In the Schwarzschild case $|\mathcal{G}|^{2} \sim M^{2} \sin ^{4}(\theta / 2)$ in the long-wavelength regime [27,28], implying a cross section of $M^{2}$ at $\theta=180^{\circ}$. In the Kerr case, the backscattered flux may be greatly enhanced by superradiance, by a factor of up to $~ 35$ times (cf. Fig. 14 in Ref. [29]). Below we show that a similar effect occurs for the scattering of purely EM waves by a ReissnerNordström black hole.

\section{ANALYSIS}

Electromagnetic and gravitational perturbations in Reissner-Nordström spacetime consist of axial (odd-parity) and polar (even-parity) modes [12-14]. The governing equations can be separated by parity $\mathcal{P}= \pm$, with + and denoting even and odd cases, respectively, leading to decoupled ordinary differential equations [12,14]

$$
\frac{d^{2}}{d r_{*}^{2}} \varphi_{ \pm}^{\mathcal{P}}+\left(\omega^{2}-V_{ \pm}^{\mathcal{P}}\right) \varphi_{ \pm}^{\mathcal{P}}=0
$$

where $r_{*}$ is the Wheeler tortoise coordinate defined by $d r / d r_{*}=f$. The \pm signs appearing in subscripts in Eq. (4) are related to the different expressions of the effective potentials $V_{ \pm}^{\mathcal{P}}$, which are explicitly given in Refs. [15,17].
We note that the modes $\varphi_{+}^{\mathcal{P}}$ exist for $l \geq 1$, and the modes $\varphi_{-}^{\mathcal{P}}$ exist for $l \geq 2$.

Radial functions for the EM and gravitational waves, $F^{\mathcal{P}}$ and $G^{\mathcal{P}}$, respectively, are given by

$$
\begin{aligned}
& F^{\mathcal{P}}=\varphi_{+}^{\mathcal{P}} \cos \psi-\varphi_{-}^{\mathcal{P}} \sin \psi, \\
& G^{\mathcal{P}}=\varphi_{-}^{\mathcal{P}} \cos \psi+\varphi_{+}^{\mathcal{P}} \sin \psi,
\end{aligned}
$$

where

$$
\sin (2 \psi)=-2 \mathcal{P} Q \frac{[(l-1)(l+2)]^{1 / 2}}{\Omega}, \quad|\psi|<\frac{\pi}{4}
$$

and

$$
\Omega=\sqrt{9 M^{2}+4 Q^{2}(l-1)(l+2)} .
$$

The scattering of a pure EM wave corresponds to the asymptotic conditions (as $r_{*} \rightarrow \infty$ )

$$
\begin{gathered}
F\left(r_{*}\right) \approx F_{\omega l}^{\mathcal{P} \text { in }} e^{-i \omega r_{*}}+F_{\omega l}^{\mathcal{P} \text { out }} e^{i \omega r_{*}}, \\
G\left(r_{*}\right) \approx G_{\omega l}^{\mathcal{P} \text { out }} e^{i \omega r_{*}},
\end{gathered}
$$

where $F_{\omega l}^{\mathcal{P} \text { in }}$, etc., are complex constants. The radial functions $\varphi_{ \pm}^{\mathcal{P}}$ have asymptotic forms

$$
\varphi_{ \pm}^{\mathcal{P}}\left(r_{*}\right) \sim\left\{\begin{array}{ll}
e^{-i \omega r_{*}}+A_{ \pm, \omega l}^{\mathcal{P}} e^{i \omega r_{*}} & \left(r_{*} \rightarrow \infty\right) \\
B_{ \pm, \omega l}^{\mathcal{P}} e^{-i \omega r_{*}} & \left(r_{*} \rightarrow-\infty\right)
\end{array},\right.
$$

which lead to

$$
\begin{gathered}
R_{\omega l}^{\mathcal{P}} \equiv \frac{F_{\omega l}^{\mathcal{P} \text { out }}}{F_{\omega l}^{\mathcal{P i n}}}=A_{+, \omega l}^{\mathcal{P}} \cos ^{2} \psi+A_{-, \omega l}^{\mathcal{P}} \sin ^{2} \psi, \\
C_{\omega l}^{\mathcal{P}} \equiv \frac{G_{\omega l}^{\mathcal{P} \text { out }}}{F_{\omega l}^{\mathcal{P i n}}}=\frac{\sin (2 \psi)}{2}\left(A_{+, \omega l}^{\mathcal{P}}-A_{-, \omega l}^{\mathcal{P}}\right) .
\end{gathered}
$$

Here, $\left|R_{\omega l}^{\mathcal{P}}\right|^{2}$ and $\left|C_{\omega l}^{\mathcal{P}}\right|^{2}$ represent the amounts of reflected (nonconverted) and converted energy, respectively, when the incident wave is purely EM. The polar and axial coefficients are related by [19]

$$
\frac{A_{ \pm, \omega l}^{+}}{A_{ \pm, \omega l}^{-}}=\frac{(l-1) l(l+1)(l+2)+2 i \omega \nu_{\mp}}{(l-1) l(l+1)(l+2)-2 i \omega \nu_{\mp}},
$$

where $\nu_{ \pm}=3 M \pm \Omega$.

The EM differential scattering cross section in spherically symmetric spacetimes (for a circularly polarized incident planar wave) was found by Fabbri [30] 


$$
\begin{aligned}
\frac{d \sigma}{d \Omega}= & \frac{1}{8 \omega^{2}}\left\{\left|\sum_{l=1}^{\infty} \frac{2 l+1}{l(l+1)}\left[e^{2 i \delta_{l}^{-}(\omega)} T_{l}(\theta)+e^{2 i \delta_{l}^{+}(\omega)} \pi_{l}(\theta)\right]\right|^{2}\right. \\
& \left.+\left|\sum_{l=1}^{\infty} \frac{2 l+1}{l(l+1)}\left[e^{2 i \delta_{l}^{-}(\omega)} \pi_{l}(\theta)+e^{2 i \delta_{l}^{+}(\omega)} T_{l}(\theta)\right]\right|^{2}\right\},
\end{aligned}
$$

with the phase shifts given by

$$
e^{2 i \delta_{l}^{\mathcal{P}}(\omega)}=(-1)^{l+1} R_{\omega l}^{\mathcal{P}}
$$

and angular functions given by

$$
\pi_{l}(\theta) \equiv \frac{P_{l}^{1}(\cos \theta)}{\sin \theta}, \quad T_{l}(\theta) \equiv \frac{d}{d \theta} P_{l}^{1}(\cos \theta),
$$

where $P_{l}^{m}(\cos \theta)$ are associated Legendre functions. Equation (15) may be recast as $\frac{1}{2}\left(|\mathcal{F}+\mathcal{G}|^{2}+|\mathcal{F}-\mathcal{G}|^{2}\right)=$ $|\mathcal{F}|^{2}+|\mathcal{G}|^{2}$, with

$$
\begin{aligned}
\mathcal{F}(\theta)= & \frac{\pi}{i \omega} \sum_{l=1}^{\infty} \sum_{\mathcal{P}= \pm}\left[\exp \left(2 i \delta_{l}^{\mathcal{P}}\right)-1\right]_{-1} Y_{l}^{1}(1)_{-1} Y_{l}^{1}(\cos \theta) \\
\mathcal{G}(\theta)= & \frac{\pi}{i \omega} \sum_{l=1}^{\infty} \sum_{\mathcal{P}= \pm}\left[\exp \left(2 i \delta_{l}^{\mathcal{P}}\right)-1\right] \mathcal{P}(-1)^{l} \\
& \times{ }_{-1} Y_{l}^{1}(1)_{-1} Y_{l}^{1}(-\cos \theta) .
\end{aligned}
$$

Here, ${ }_{s} Y_{l}^{m}(\cdot)$ are the spin-weighted spherical harmonics [31], and $\mathcal{F}$ and $\mathcal{G}$ are the helicity-preserving and helicityreversing amplitudes. A consequence of Eq. (14) is that $\delta_{l}^{+} \neq \delta_{l}^{-}$, except for in the Schwarzschild case; hence $\mathcal{G} \neq 0$ for charged black holes. We note that $\mathcal{F}\left(\theta=180^{\circ}\right)=0$ by construction, whereas $\mathcal{G}\left(\theta=180^{\circ}\right) \neq 0$.
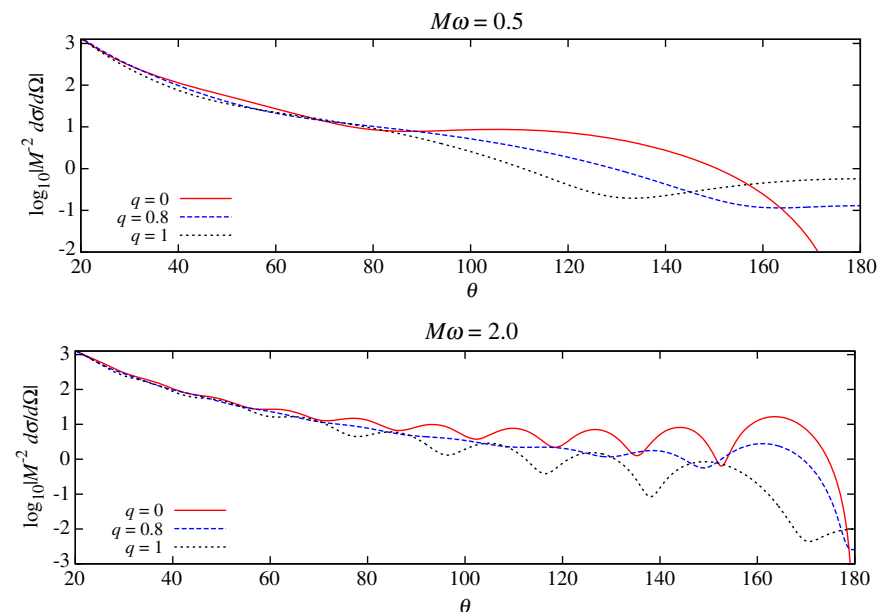

By applying the method of Ref. [28] we may obtain approximations for $\mathcal{F}$ and $\mathcal{G}$ in the low-frequency regime. We find

$$
|\mathcal{G}|^{2}=q^{4} M^{2} \sin ^{4}(\theta / 2)+O\left(\omega^{2}\right)
$$

with $q \equiv|Q| / M$. At low frequencies the only contribution to $\mathcal{G}$ is from the $l=1$ mode. This shows that helicity reversal and electromagnetic-to-gravitational conversion are distinct phenomena, as the latter occurs only for $l \geq 2$.

To compute the cross section for general frequencies, we used numerical methods. First, we computed the phase shifts by solving the radial equations numerically and matching the solutions onto their analytical asymptotic forms. Second, we used a convergence method to sum the (formally divergent) partial-wave series (15).

For the first step, the asymptotic forms (11) are not sufficiently accurate. Instead, we write the asymptotic form of the solutions to the radial equations (4) in terms of spherical Hankel functions $h_{l}^{(1)}$, which are obtained by keeping only the term $l(l+1) / r^{2}$ in the effective potentials of Eq. (4). For the second step, we adapted the method first introduced by Yennie et al. [32], which has been successfully applied to e.g. the study of scalar scattering by Reissner-Nordström black holes [20].

Figure 1 shows the scattering cross sections obtained numerically for the cases $q=0,0.8,1$ and $M \omega=0.5,1,2,3$. Increasing the charge-to-mass ratio $q$ at fixed $M \omega$ leads to wider "orbiting" oscillations, and a smaller (average) flux at large angles.

A novel feature of scattering of EM waves by ReissnerNordström black holes is the appearance of flux on axis in the backward direction for $0<q \leq 1$. This effect, although small, can be seen clearly in Fig. 1. Figure 2 illustrates the angular profile of this flux, as it might be seen in a detector. A bright spot of flux around $180^{\circ}$ in the extreme case (right) contrasts with the dark spot in the Schwarzschild case (left).
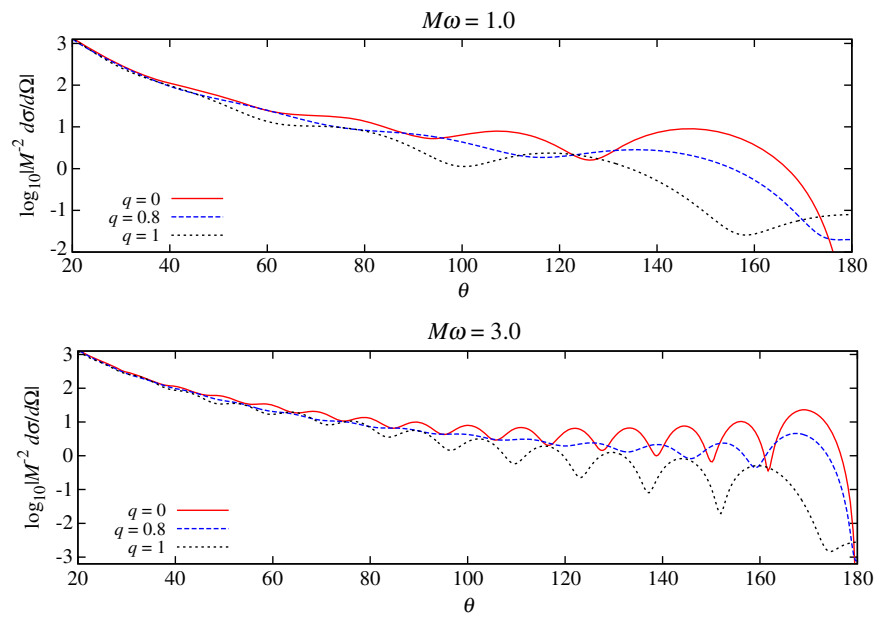

FIG. 1 (color online). Electromagnetic scattering by Reissner-Nordström black holes for $q=0,0.8,1$ and $M \omega=0.5,1.0,2.0,3.0$. For $0<q \leq 1$, the flux of EM radiation in the backward direction is nonzero; it diminishes as $M \omega$ increases. 

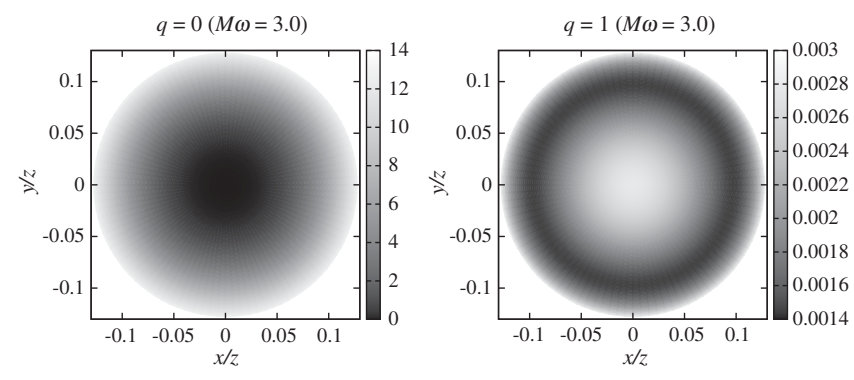

FIG. 2. Illustration of an electromagnetic-wave detection in the backward direction for a Schwarzschild (left) and an extreme Reissner-Nordström (right) black hole. Here, $M \omega=3.0$ and the scattering angle interval is between $172.6^{\circ}$ and $180^{\circ}$ in both graphs.

\section{DISCUSSION}

We have explored a novel signature of black hole charge: the nonzero flux around $180^{\circ}$ when a black hole scatters EM plane waves. We have shown that this effect arises from parity dependence in the scattering phase shifts, leading to a helicity-reversed scattering amplitude $\mathcal{G}(\theta)$. At low frequencies, the helicity-reversed flux is primarily in the $l=1$ mode, and the cross section at $180^{\circ}$ is $\sim Q^{4} / M^{2}$ [cf. Eq. (19)]. Note that the backscattered flux is dissimilar to a backward glory, as it diminishes as $M \omega$ is increased and is primarily a low-frequency, low-multipole effect.

Parity dependence in scattering is related to the presence of a source for the field. On one hand, the black hole's mass acts as a source for the gravitational field, leading to parity dependence in the gravitational sector. Parity dependence for gravitational waves on uncharged spacetimes was noted many years ago, and was shown to lead to nonzero backscattering in Refs. [33-35]. On the other hand, the black hole's charge acts as a source for the EM field, generating parity dependence and backscattering in the EM sector. In the low-frequency limit the helicity-reversed cross sections take a similar form, with $|\mathcal{G}|^{2} \sim M^{2} \sin ^{4}(\theta / 2)$ and $|\mathcal{G}|^{2} \sim q^{4} M^{2} \sin ^{4}(\theta / 2)$ in the gravitational and EM cases, respectively; in the extremal limit $q \rightarrow 1$ these become identical.

We note that helicity reversal and electromagnetic-togravitational conversion are two rather distinct effects. The former arises from parity dependence as exhibited by Eq. (14). The latter arises from mixing between EM and gravitational sectors, via Eq. (7). The latter, conversion, will generate other interesting effects in scattering, such as the generation of EM flux from gravitational-wave irradiation, and vice versa.

In principle, as described here, observations of backscattered EM flux allow one to infer the black hole's charge. In practice, the backscattering effect is likely to be negligible in astrophysical black hole scenarios due to its low-frequency character. In this regard it is somewhat like the Hawking effect. On the other hand, it is known that, for a rapidly spinning black hole, the backscattering of gravitational waves is greatly enhanced by superradiance [29]. We anticipate that EM backscattering will be similarly enhanced in the Kerr-Newman black hole case. Thus, we conclude that the backscattering effect is an interesting aspect of black hole phenomenology that warrants further investigation.

\section{ACKNOWLEDGMENTS}

The authors are grateful to Conselho Nacional de Desenvolvimento Científico e Tecnológico (CNPq) and to Coordenação de Aperfeiçoamento de Pessoal de Nível Superior (CAPES) for partial financial support. A. H. and L.C.B.C. also acknowledge partial support from the Abdus Salam International Centre for Theoretical Physics through the Visiting Scholar/Consultant Programme and Associates Scheme, respectively. A. H. and S.R.D. thank the Universidade Federal do Pará (UFPA) in Belém for kind hospitality.
[1] H. Geiger and E. Marsden, Proc. R. Soc. A 82, 495 (1909).

[2] E. Rutherford, in Background to Modern Science: Ten Lectures at Cambridge Arranged by the History of Science Committee 1936, edited by J. Needham and W. Pagel (Cambridge University Press, Cambridge, England, 1938).

[3] A. Einstein, Science 84, 506 (1936).

[4] S. Chandrasekhar and S. Detweiler, Proc. R. Soc. A 344, 441 (1975).

[5] R. A. Matzner, C. DeWitt-Morette, B. Nelson, and T.-R. Zhang, Phys. Rev. D 31, 1869 (1985).
[6] J. A. H. Futterman, F. A. Handler, and R. A. Matzner, Scattering from Black Holes (Cambridge University Press, Cambridge, England, 1988).

[7] A. A. Starobinsky, Sov. Phys. JETP 37, 28 (1973).

[8] B. Mashhoon, Phys. Rev. D 10, 1059 (1974).

[9] S. W. Hawking, Nature (London) 248, 30 (1974); Commun. Math. Phys. 43, 199 (1975).

[10] F. J. Zerilli, Phys. Rev. D 9, 860 (1974).

[11] U. H. Gerlach, Phys. Rev. Lett. 32, 1023 (1974).

[12] V. Moncrief, Phys. Rev. D 9, 2707 (1974); 10, 1057 (1974); 12, 1526 (1975). 
[13] D. W. Olson and W. G. Unruh, Phys. Rev. Lett. 33, 1116 (1974).

[14] R. A. Matzner, Phys. Rev. D 14, 3274 (1976).

[15] L. C. B. Crispino, A. Higuchi, and E. S. Oliveira, Phys. Rev. D 80, 104026 (2009).

[16] L. C. B. Crispino, A. Higuchi, and G. E. A. Matsas, Phys. Rev. D 82, 124038 (2010).

[17] E. S. Oliveira, L. C. B. Crispino, and A. Higuchi, Phys. Rev. D 84, 084048 (2011).

[18] L. C. B. Crispino, S. R. Dolan, and E. S. Oliveira, Phys. Rev. Lett. 102, 231103 (2009).

[19] S. Chandrasekhar, The Mathematical Theory of Black Holes (Oxford University Press, New York, 1983).

[20] L. C. B. Crispino, S. R. Dolan, and E. S. Oliveira, Phys. Rev. D 79, 064022 (2009).

[21] E. F. Eiroa, G. E. Romero, and D. F. Torres, Phys. Rev. D 66, 024010 (2002).

[22] A. Bhadra, Phys. Rev. D 67, 103009 (2003).

[23] M. Sereno, Phys. Rev. D 69, 023002 (2004).
[24] P. Anninos, C. DeWitt-Morette, R. A. Matzner, P. Yioutas, and T. R. Zhang, Phys. Rev. D 46, 4477 (1992).

[25] T.-R. Zhang and C. DeWitt-Morette, Phys. Rev. Lett. 52, 2313 (1984).

[26] P. L. Chrzanowski, R. A. Matzner, V. D. Sandberg, and M. P. Ryan, Phys. Rev. D 14, 317 (1976).

[27] R. A. Matzner and M. P. Ryan, Phys. Rev. D 16, 1636 (1977).

[28] S. R. Dolan, Phys. Rev. D 77, 044004 (2008).

[29] S. R. Dolan, Classical Quantum Gravity 25, 235002 (2008).

[30] R. Fabbri, Phys. Rev. D 12, 933 (1975).

[31] J. Goldberg, A. Macfarlane, E. Newman, F. Rohrlich, and E. Sudarshan, J. Math. Phys. (N.Y.) 8, 2155 (1967).

[32] D. R. Yennie, D. R. Ravenhall, and R. N. Wilson, Phys. Rev. 95, 500 (1954).

[33] P. J. Westervelt, Phys. Rev. D 3, 2319 (1971).

[34] P. C. Peters, Phys. Rev. D 13, 775 (1976).

[35] W. K. De Logi and S. J. Kovács, Phys. Rev. D 16, 237 (1977). 